\title{
Légitimer les experts religieux, sous la République romaine
}

Yann BERTHELET *

Alors que les prêtres publics de la République romaine ne recevaient aucune formation spécifique avant leur accès à un sacerdoce ${ }^{1}$, au point que certains historiens sont allés jusqu'à les qualifier d' «amateurs », ils étaient cependant considérés par leurs contemporains comme des experts religieux. Souvent présentés comme détenteurs d' " expérience " (peritia), de " science » (scientia, sapientia) et d' "autorité" (auctoritas) ${ }^{3}$, ils étaient régulièrement consultés par le Sénat, les magistrats ou les particuliers ${ }^{4}$, accomplissaient

\footnotetext{
* Doctorant à l'Université Paris 1 - Panthéon-Sorbonne, il prépare, sous la direction de Jean-Michel David et John Scheid, une thèse intitulée Gouverner par les signes divins : recherches sur l'autorité divinatoire publique, sous la République romaine.

1. M. BEARD, "Priesthood in the roman republic», dans Pagan Priests. Religion and Power in Ancient World, M. BEARD et J. NORTH éd., Londres, 1990, p. 36; J. RÜPKE, "Controllers and professionals: analyzing religious specialists", Numen, 43/3 (1996), p. 252 (repris dans "Fasti sacerdotum". Die Mitglieder der Priesterschaften und das sakrale Funktionspersonal römischer, griechischer, orientalischer und jüdisch-christlicher Kulte in der Stadt Rom von 300 v. Chr. bis 499 n. Chr., Stuttgart, 2005, t. III, p. 1414 (PAwB, 12)) ; J. SCHEID, Religion et piété à Rome, Paris, 20012, p. 54 ; ID., La Religion des Romains, Paris, 2005', p. 111. Il en va autrement des haruspices étrusques (M.-L. HAACK, Les Haruspices dans le monde romain, Bordeaux, 2003, p. 41-43).

2. Voir, par exemple, H. H. SCULlaRD, Festivals and Ceremonies of the Roman Republic, Londres, 1981, p. 28.

3. Dans le De domo de Cicéron, nous trouvons, appliqués aux pontifes, les termes sapientia (1,2 et 45), scientia (117, 118, 138 et 139), scire (33), auctor-auctoritas (2, 44, 45, 69, 100, 104, 117, 118, 120, 132, 137, 139, 142 et 147), peritus (118), peritiores (117), peritissimus (139).

4. Cicéron, De legibus, G. DE Plinval trad., Paris, 1959 (Collection des universités de France) (désormais Cic., Leg.), 2, 20 : «Que ceux qui ne savent pas s'instruisent auprès des prêtres de l'État»; 2, 29: "Des prêtres en certain nombre [...] fournissent le
} 
eux-mêmes certains rites publics ou, le plus souvent, assistaient dans cette tâche les magistrats. Ces derniers apparaissent en effet dans les sources comme les principaux officiants du culte, avec l'assistance et sous le contrôle des prêtres-experts ${ }^{5}$.

Ce paradoxe d'experts religieux sans formation initiale suppose que la société romaine reconnaissait, d'une manière ou d'une autre, leur légitimité. Il reste à comprendre selon quels procédés. L'acquisition de savoirs et de savoir-faire après l'entrée dans un collège sacerdotal était-elle déterminante ? Quel rôle convient-il d'attribuer aux pratiques du secret et de la collégialité, caractéristiques de l'expertise sacerdotale ? Quant aux rapports étroits que les prêtres romains entretenaient avec le pouvoir politique, doit-on considérer qu'ils renforçaient ou, au contraire, fragilisaient leur légitimité d'experts ?

Abusés par l'évocation, dans les sources, de livres sacerdotaux, de nombreux chercheurs ont formulé l'hypothèse de Ritualbücher normatifs, dans lesquels les prêtres auraient pu acquérir leur savoir ${ }^{6}$. John Scheid a toutefois montré que les livres sacerdotaux contenaient seulement "la jurisprudence sacrée et les comptes rendus des rites célébrés. Les données étaient enregistrées sous forme historique avec un grand soin du détail, comme il sied pour un document public. À Rome, la tradition repose sur ce type de documents, et non sur des normes abstraites et exhaustives, figurant dans des Ritualbücher généraux " ${ }^{7}$. Il convient donc de concevoir une tradition religieuse romaine essentiellement orale, qui se transmettait en partie "avant " l'entrée dans la vie publique. Lorsqu'il n'était encore qu'un jeune aristocrate, le futur homme public acquérait auprès de son père ou d'un autre parent les savoir-faire élémentaires du culte, tels les rituels du sacrifice ou de la consultation des dieux. Débutant ensuite dans un

moyen de répondre aux consultations de droit (religieux) et d'exécuter les cérémonies liturgiques".

5. J. SCHEID, «Les activités religieuses des magistrats romains", dans Herrschen und Verwalten. Der Alltag der römischen Administration in der Hohen Kaiserzeit, R. HAENSCH et J. HEINRICHS éd., Cologne/Weimar/Vienne, 2007, p. 143-144 ; J. RÜPKE, " Controllers and professionals », art. cité,, p. 252, repris dans « Fasti sacerdotum »..., op. cit., p. 1414 ; J.-C. LACAM, Variations rituelles. Les pratiques religieuses en Italie centrale et méridionale au temps de la denxième guerre punique, Rome, 2010 (Collection de l'École française de Rome, 430), p. 101-109.

6. Pour la bibliographie, voir J. SCHEID, «Les archives de la piété. Réflexions sur les Livres sacerdotaux ", dans La Mémoire perdue. À la recherche des archives oubliées, publiques et privées, de la Rome antique, S. DEMOUGIN éd., Paris, 1994, p. 174-176 et 185.

7. J. SCHEID, «"Livres sacerdotaux" et érudition : l'exemple des chapelles des Argées", dans Zwischen Krise und Altag. Conflit et normalité, C. BATSCH, U. EGELHAAF-GAISER et R. STEPPER éd., Stuttgart, 1999, p. 161 (PAwB, 1). 
sacerdoce - ou une magistrature -, il apprenait le détail des rites propres à sa fonction auprès de ses collègues, des appariteurs et des esclaves publics qui l'assistaient. Il ne s'appuyait sur l'écrit que pour la récitation des formules, des prières ou des hymnes, qui possédaient un caractère immédiatement performatif ${ }^{8}$.

L'absence de formation préalable obligeait les plus jeunes dans le sacerdoce à se reposer sur leurs appariteurs, déplaçant ainsi sur eux la responsabilité des savoirs et des savoir-faire sacerdotaux. Or Cicéron - ou du moins son personnage - souligne, dans le traité Sur la divination, que l'assistant religieux', jadis un expert (peritus), pouvait à son époque être n'importe qui (quilubet) ${ }^{10}$. Il arrivait même qu'un jeune prêtre dût assister seul, sans aucune aide, un magistrat dans l'accomplissement d'un rite. Contestant la validité de la consécration de sa maison à la déesse Libertas par Clodius, Cicéron met ainsi en cause l'isolement du jeune pontife inexpérimenté, Lucius Pinarius Natta, qui assista le tribun :

"Qui donc fut présent ? "Le frère de ma femme, dit [Clodius]." Si nous cherchons l'autorité, il est d'un âge à ne pas en avoir encore, et, quelle que puisse être l'autorité d'un jeune homme, une si étroite parenté ne peut que l'affaiblir. Mais, si on cherche le savoir [sin autem scientia est quasita], qui avait moins d'expérience [quis erat minus peritus] que celui qui était entré au collège peu de jours auparavant ? [...] un adolescent inexpérimenté [imperitus], un prêtre novice, cédant aux prières d'une sœur et aux menaces d'une mère, agissant dans l'ignorance [ignarus] et à contrecœur, sans collègues et sans livres, sans personne pour l'inspirer ou l'assister $[\ldots]{ }^{11}$

L'absence de formation spécifique ne portait pas à conséquence uniquement pour les jeunes prêtres. Ainsi, Tiberius Sempronius Gracchus, le père des Gracques, pourtant augure depuis plus de quarante ans, commit par ignorance, lors de son second consulat en 163 av. J.-C., une erreur sur les auspices. S'il fit cette erreur en tant que magistrat et non en tant que prêtre, il reste significatif, pour mon propos, qu'il ne prît conscience de son erreur

8. J. SCHEID, "Oral tradition and written tradition in the formation of sacred law in Rome ", dans Religion and Law in Classical and Christian Rome, C. ANDO et J. RÜPKE éd., Stuttgart, 2006 (PAwB, 15), p. 17-18.

9. Cicéron parle du pullarius, appariteur des magistrats pour la prise d'auspices ex tripudiis. Mais la remarque vaut sans doute aussi pour les appariteurs de prêtres.

10. Cicéron, De divinatione, G. FreYBurGer et J. SCHeID trad., 1992 (désormais Cic., Div.), 2, 71.

11. Cicéron, De domo, P. Wuilleumier trad., Paris, 1952 (Collection des université de France) (désormais Cic., Dom.), 118 et 139. Cf. ibid., 135. 
qu'après avoir consulté les livres auguraux ${ }^{12}$. En dépit de ces constats d'ignorance chez ceux qui avaient la charge des cultes publics, on peut toutefois penser que les magistrats et les prêtres romains maîtrisaient, par expérience aristocratique et transmission orale, les pré-requis nécessaires à l'accomplissement des rites. Lorsque des erreurs étaient commises, il était en outre toujours possible de répéter le rite par son instauratio.

Accomplir des rites publics ou, bien plus souvent, assister le magistrat dans cette tâche, n'épuisait toutefois pas les fonctions sacerdotales: les prêtres romains devaient aussi répondre aux questions des sénateurs, des magistrats ou des particuliers en matière de droit sacré, ce qui fait problème lorsqu'on sait qu'ils ne recevaient aucune formation préalable en la matière. Cette difficulté n'échappait pas aux Anciens, qui avaient parfaitement conscience que tous leurs prêtres n'étaient pas aussi compétents en matière de droit pontifical ou augural : ils reconnaissaient que certains s'y étaient plus particulièrement spécialisés, sans doute après leur entrée dans le sacerdoce $^{13}$. Ainsi en est-il de plusieurs pontifes, tel Coruncanius, dont Cicéron dit qu'il "passe pour le plus savant des pontifes [peritissimus pontifex] » ou tels les deux Scaevola, qualifiés de "très compétents en droit [iuris peritissimi]». La compétence juridique que Cicéron vante ici ne se limitait d'ailleurs pas au droit pontifical mais s'étendait au droit civil: Publius Mucius Scaevola disait souvent "qu'on ne pouvait être bon pontife sans connaître le droit civil ${ }^{14}$. Pour les augures, il faut citer Appius Claudius Pulcher, dont le personnage de Quintus oppose, dans le traité Sur la divination, la science augurale et divinatoire à l'ignorance de ses collègues : "J'approuve vivement cet homme, qui est le seul, depuis de longues années à avoir conservé la science non seulement des formules augurales [memoria augurii], mais encore de la divination [divinandi disciplina]. Tes collègues se moquaient de lui [...] ${ }^{15}$. Mais un passage du Brutus de Cicéron nous rappelle que sa spécialisation en droit augural n'était que relative: Appius Claudius était " un orateur assez diligent, qui avait beaucoup de savoir et de pratique; il possédait en outre, avec le droit augural, la science [bene peritus fuit] de tout le droit public et l'histoire de notre passé ${ }^{16}$. Comme tout aristocrate, il devait connaître le droit public en

12. Cicéron, De natura deorum (désormais Cic., Nat. deor.), 2, 10-11. Cf. ID., Div., 1, 33.

13. J. RÜPKE, «Controllers and professionals », art. cité., p. 255, repris dans « Fasti sacerdotum »..., op. cit., p. 1416.

14. Cic., Leg., 2, 47.

15. Cic., Div., 1, 105.

16. Cicéron, Brutus, J. MARTHA trad., Paris, 1973 (Collection des universités de France), 267. 
général et maîtriser l'art oratoire afin de pouvoir défendre ses clients, attaquer ses adversaires et parler en public; et avoir suffisamment d'expérience militaire pour commander des troupes en tant que consul ou promagistrat $^{17}$.

Quintus n'est pas le seul à regretter que la science augurale soit ignorée par les augures eux-mêmes. Dans le traité de Cicéron Sur la nature des dieux, le personnage du stoïcien Balbus affirme ainsi que "par la négligence des grandes familles [neglegentia nobilitatis], la science augurale s'est perdue [augurii disciplina omissa] " ${ }^{18}$. On lit de même dans son traité Sur les lois: «il est incontestable que la théorie et la pratique de l'art augural se sont évanouies du fait de l'ancienneté et de la négligence [uetustate et neglegentia] " ${ }^{19}$. Caton le Censeur aurait quant à lui déploré qu'un grand nombre d'auspices aient été " complètement perdus et délaissés [amissa plane et deserta] par la négligence du collège des augures [neglegentia collegii] ${ }^{20}$. En plein Sénat, le futur triumvir Marc-Antoine, augure depuis 50 av. J.-C., vit sa compétence violemment mise en doute par Cicéron, son collègue à l'augurat :

« [Antoine,] cet excellent augure déclara que le sacerdoce qu'il exerçait lui donnait, par les auspices, le pouvoir d'interdire la réunion des comices ou de les frapper de nullité, et il assura qu'il allait agir ainsi [...] Admettons que ce soit ignorance [imperite] [...] Mais ici, l'ignorance [inscientia] se complique de malhonnêteté, car il ignore ce qu'un augure doit savoir et ne fait pas ce qui convient à un homme d'honneur. " ${ }^{21}$

L'invective, évidemment outrancière, témoigne toutefois qu'il était possible à un personnage consulaire, lui-même augure, de formuler publiquement des doutes sur les connaissances augurales d'un des premiers personnages de la République.

Que l'ignorance des adversaires de Cicéron fût réelle ou non, le témoignage du célèbre orateur laisse peu de doutes sur l'inégale compétence des prêtres en matière de droit sacré : seules quelques figures, tels les deux Scævola ou Appius Claudius Pulcher, ont pu construire leur légitimité

17. Voir C. MOATTI, "Experts, mémoire et pouvoir à Rome, à la fin de la République ", Revue historique, 626 (2003), p. 307-311; J.-M. DAVID, «L'éloquence judiciaire entre compétence aristocratique et spécialisation carriériste", dans Von der militia equestris zur militia urbana. Der Wandel der Karrierefelder in der römischen Republik, K.-J. HÖLKESKAMP éd. (à parâtre).

18. Cic., Nat. deor., C. AUVRAY-ASSAYAS trad., Paris, 2002 (La Roue à livres), 2, 9.

19. Cic., Leg., 2, 33.

20. Cic., Div., 1, 28.

21. Cicéron, Philippiques, A. Boulanger et P. Wutlueumier trad., Paris, 1963 (Collection des universités de France) (désormais Cic., Phil.), 2, 80-81. 
d'experts religieux sur leurs seules compétences juridiques. Il convient donc de chercher d'autres procédés de légitimation, susceptibles d'opérer pour tous les prêtres.

L'un d'entre eux réside de toute évidence dans le caractère collégial de l'expertise religieuse à Rome. C'est en effet dans son ensemble que le collège sacerdotal est généralement consulté, et c'est en son nom que le décret sacerdotal est rendu. On trouve ainsi, pour la consultation des prêtres, des formules du type "le Sénat ordonna au collège des pontifes de statuer [senatus pontificum collegium consuli iussit] [...]»" et, pour la réponse, des formules du type "les pontifes décrétèrent de même que [decrevere item pontifices $u t][\ldots] "{ }^{23}$. La collégialité du responsum sacerdotal est en effet perçue comme une garantie supplémentaire de la justesse de l'expertise. Ainsi, en 200 av. J.-C., alors que le consul s'apprêtait à vouer des jeux à Jupiter, "le grand pontife Publius Licinius Crassus émit une objection en prétendant que l'État ne pouvait formuler de vœu si la somme allouée n'était pas précisée [...] Les pères furent ébranlés par l'objection et l'autorité de celui qui la présentait; ils invitèrent pourtant le consul à demander au collège des pontifes s'il était possible de formuler un vœu sans fixer la somme engagée. Les pontifes décrétèrent que c'était possible et même que cette façon de faire était plus régulière ${ }^{24}$. Or le Sénat suivit l'avis du collège.

La collégialité semble avoir été recherchée en matière de rite comme en matière de consilium. Un expert religieux restait ainsi contrôlé par ses collègues, même lorsqu'il agissait seul. Une affaire survenue dans un camp militaire romain, en 293 av. J.-C., l'illustre assez bien :

"Au camp tous sans exception brûlaient de se battre [...] Cette ardeur générale gagna à son tour ceux qui s'occupaient des auspices : comme les poulets refusaient de manger, leur gardien n'hésita pas à donner de faux auspices et annonça au consul que les poulets picoraient avec appétit [...] une discussion éclata entre les pullaires au sujet des auspices du jour. Les cavaliers l'entendirent et [...] firent savoir [...] au neveu du consul qu'il y avait contestation sur les auspices. ${ }^{25}$

Si la légitimité de l'expertise sacerdotale n'était guère affectée par les ignorances individuelles, c'est aussi parce que les prêtres étaient les seuls à avoir accès au savoir qu'ils étaient censés maîtriser. Comme tout expert (peritus), le prêtre était celui qui savait ce que personne d'autre ne pouvait

22. Tite-Live, Ab urbe condita (désormais Liv.), 39, 5, 9. Trad. pers.

23. Liv., 27, 37, 7. Trad. pers.

24. Liv., A. FlOBERT trad., Paris, 1997, 31, 9, 7-8.

25. Liv., A. FloberT trad., Paris, 1996, 10, 40, 3-9. 
savoir $^{26}$. Il en résultait un monopole des prêtres sur leur domaine d'expertise. De cette manière, les critiques relatives à leur compétence ne pouvaient être qu'internes $^{27}$. Ainsi, c'est parce qu'il est lui-même augure que Cicéron peut dénoncer publiquement la soi-disant incompétence de son collègue, l'augure Marc-Antoine. En revanche, lorsqu'il s'en prend au jeune pontife Pinarius Natta, c'est la prudence qui s'impose :

"Je ne parle ni du droit pontifical ni des formules de la consécration ellemême ni du cérémonial religieux; je ne cache pas que je les ignore, et, même si je les savais, je les cacherais, pour ne pas paraitre importun aux autres et indiscret à vous-mêmes [vobis etiam curiosus]; cependant de votre discipline transpirent maints détails, qui glissent souvent jusqu'à nos oreilles [...] J'ai dit en commençant que je ne parlerai ni de votre science, ni du rituel, ni des secrets du droit pontifical [de abscondito pontificum iure]. Ce que j'ai déclaré jusqu'ici à propos des dédicaces, je ne l'ai pas tiré de quelques archives secrètes [ex occulto aliquo genere litterarum], mais je l'ai pris dans le fonds commun, dans les actes publics des magistrats et dans leurs rapports au collège des pontifes. ${ }^{28}$

L'attachement des pontifes au secret entourant leur science est bien attesté par ailleurs. Au lendemain de l'incendie de Rome par les Gaulois en 390 av. J.-C., « les pères firent d'abord rechercher ce qui restait des traités et des lois [...] Certaines de ces lois furent même portées à la connaissance du public, mais celles qui concernaient la pratique du culte demeurèrent secrètes à la demande expresse des pontifes " ${ }^{29}$. Et la publication, en 304 av. J.-C., par Gnaeus Flavius, des fastes pontificaux ${ }^{30}$ et des formules des actions de la loi, délivrées jusque là par les pontifes, fut très clairement une attaque contre cet exclusivisme pontifical : "Il divulgua le droit public, jusque-là tenu en réserve, comme au fond d'un sanctuaire, entre les mains des pontifes [repositum in penetralibus pontificum], et il fit placer autour du Forum le tableau des jours fastes, afin que l'on sût quand il était permis de plaider $»^{31}$.

26. Cf. Cicéron, Pro Murena, 28.

27. Notons que les collèges sacerdotaux ne débattaient pas au vu et au su de tous : seule la décision finale était rendue publique, sous la forme d'un décret remis au Sénat.

28. Cic., Dom., 121 et 138 ; cf. ibid., 33.

29. Liv., A. FlOBERT trad., Paris, 1996, 6, 1, 10.

30. Sur lépisode, voir M. HumM, Appius Claudius Cacus. La République accomplie, Rome, 2005 (Bibliothèque des Écoles françaises d'Athènes et de Rome, 322), p. 451-455, avec la bibliographie antérieure.

31. Liv., 9, 46, 5. Traduction empruntée à M. HuMM, Appius Claudius Cacus..., op. cit., p. 450. 
Les livres auguraux, qualifiés à plusieurs reprises de "secrets " (reconditi) $^{32}$, ne sont pas plus accessibles que les archives pontificales ${ }^{33}$. Dans le discours Sur sa maison, Cicéron, qui n'est pas encore augure à cette date, se montre tout aussi prudent que pour le droit pontifical : "J'en arrive aux augures; sans fouiller dans leurs livres sacrés, sans avoir l'indiscrétion [curiosus] d'approfondir le droit augural, je sais ce que j'ai appris avec le peuple, ce qui a été souvent répondu dans les assemblées " ${ }^{34}$. Les Livres sibyllins sont eux aussi accessibles aux seuls (quin)décemvirs sacris faciundis ${ }^{35}$.

Le contrôle de l'expert par ses pairs et le monopole des prêtres sur leur domaine d'expertise, dont je viens de mettre en évidence l'effet légitimant, relèvent en fait tous deux d'un seul et même processus de "professionnalisation " ${ }^{36}$.

Le processus de construction d'une légitimité expertale ne saurait cependant se limiter à la détention, collégiale et secrète, de compétences réelles ou supposées. Il faut aussi prendre en compte le rapport que les experts entretiennent au pouvoir. C'est particulièrement vrai pour les prêtres romains, dont la proximité au pouvoir politique, loin d'être un obstacle à leur légitimation, en est au contraire un des facteurs décisifs.

De fait, les détenteurs des sacerdoces publics étaient aussi, souvent, ceux qui détenaient le pouvoir politique ${ }^{37}$. Ce cumul des charges politiques et sacerdotales par les mêmes individus n'impliquait pas, toutefois, de confusion entre les fonctions $^{38}$. Un passage de la Correspondance de Cicéron,

32. Cic., Dom., 39 ; Servius auctus, In Vergilii Aeneidos libros commentarius, 1, 398 ; 2, 649.

33. J. LINDERSKI, "The Libri reconditi», dans Roman Questions. Selected Papers, Stuttgart, 1995 (HABES, 20), p. 496-523.

34. Cic., Dom., 39.

35. Cf. Cic., Div., 2, 112 ; Leg., 2, 30. Voir J. SCHEID, "Les Livres sibyllins et les archives des quindécemvirs ", dans La Mémoire perdue. Recherches sur l'administration romaine, C. NiCOLET, C. MOATTI et J. SCHEID éd., Rome, 1998 (Collection de l'École française de Rome, 243), p. 11-26.

36. J. RÜPKE, "Controllers and professionals", art. cité, p. 255-258, repris dans " Fasti sacerdotum "..., op. cit., p. 1417-1418.

37. Voir G. J. SZEMLER, The Priests of the Roman Republic. A Study of Interactions between Priesthoods and Magistracies, Bruxelles, 1972 (Latomus, 127), p. 189-191; J. SCHEID, « Le prêtre et le magistrat. Réflexions sur les sacerdoces et le droit public à la fin de la République », dans Des ordres à Rome, C. NiCOLET éd., Paris, 1984, p. 248-266; F. VAN HAEPEREN, Le College pontifical (3 s. a. C. 4 s. p. C.). Contribution à l'étude de la religion publique romaine, Bruxelles/Rome, 2002, p. 111.

38. J. BLEICKEN, "Kollisionen zwischen sacrum und publicum, eine Studie zum Verfall der altrömischen Religion ", Hermès, 85 (1957), p. 446-480. 
relatif à l'affaire de sa maison palatine, sur laquelle les pontifes venaient de délibérer, est très clair à cet égard :

"Le Sénat tient séance au complet. Il y a là tous les pontifes qui étaient sénateurs. Marcellinus, qui m'était très favorable, appelé à donner son avis le premier, leur demanda quel principe les avait guidés quand ils avaient pris leur décret. Alors Marcus Lucullus, interprète de tous ses collègues, répondit [...] [qu'ils] avaient statué sur l'aspect religieux du problème [de religione]; ils statueraient au Sénat avec le Sénat sur son aspect juridique [de lege]. "

En outre, les responsa ou decreta des prêtres relevaient strictement du consilium. Pour qu'une décision sacerdotale puisse devenir exécutoire, elle devait être confirmée par un sénatus-consulte, puis promulguée par un édit de magistrat, comme dans l'exemple suivant: "Toujours sur proposition [des décemvirs sacris faciundis], un décret du Sénat et un édit des consuls ordonnèrent trois jours de prière et de fêtes religieuses dans toute l'Italie ${ }^{40}$.

Aussi, loin de jeter le doute sur l'indépendance de l'expertise religieuse par rapport au pouvoir politique, le cumul des deux fonctions par les mêmes hommes est au contraire perçu comme une garantie de la bonne gestion à la fois des cultes et du gouvernement :

«Parmi les nombreuses créations et institutions que les dieux ont inspirées à nos ancêtres, pontifes, il n'en est pas de plus belle que leur décision de confier aux mêmes hommes le culte des dieux immortels et les intérêts supérieurs de l'État, afin que les citoyens les plus éminents et les plus illustres assurent le maintien du culte par une bonne gestion de l'État et celui de l'État par une sage exégèse du culte. " ${ }^{41}$

Cette captatio benevolentic du discours de Cicéron Sur sa Maison, prononcé devant le collège des pontifes, n'est certes pas dénuée de flatterie, mais reflète cependant une conception de la cité partagée par Cicéron et ses auditeurs, celle d'une "cité commune des dieux et des hommes [civitas communis deorum atque hominum] ${ }^{42}$ : gouverner la cité, c'est aussi prendre soin des relations avec les concitoyens supérieurs et autres que sont les dieux.

Il ne faudrait pas oublier, enfin, l'effet légitimant du processus même de désignation des prêtres, sur lequel il serait toutefois trop long de revenir en détail dans le cadre restreint de cette communication. Rappelons

39. Cicéron, Lettres à Atticus, L.-A. Constans trad., Paris, 1935 (Collection des universités de France, Correspondance, t. II, p. 98), 4, 2, 3-4.

40. Liv., C. GouillarT trad., Paris, 1986 (Collection des universités de France), 40, 19, 5. Voir J. SCHEID, Religion et Piété..., op. cit., p. 67.

41. Cic., Dom., 1.

42. Cic., Leg., 1, 23. Cf. ID., Nat. deor., 2, 154. 
simplement que les prêtres étaient désignés par une procédure de cooptation, l'expert désignant l'expert. Au cours des III et II siècles av. J.-C., cette cooptation fut doublée d'une élection restreinte, par 17 tribus. Les principaux prêtres étaient ensuite "inaugurés " ${ }^{43}$, les augures demandant à la divinité s'il était conforme ou non à l'ordre divin (fas ou nefas) que l'individu coopté par le collège devînt effectivement prêtre. La légitimation humaine était ainsi doublée d'une légitimation divine, dont il ne faudrait pas sous-estimer l'importance.

Précisons un peu, pour conclure, le profil des experts abordés dans cette étude. Nous y invite un article de Claudia Moatti, où il est montré que l'expertise avait peu de place à Rome avant le II siècle av. J.-C. ${ }^{44}$, moment de transformations considérables dans la société romaine, moment de crise culturelle et de crise des autorités traditionnelles, qui entraîna un besoin de clarification et de refondation, y compris dans le domaine intellectuel. S'y opéra en effet un double mouvement de spécialisation et d'organisation des savoirs, qui permit l'émergence d'une figure autonome de l'expert savant, distincte de celle du gouvernant ${ }^{45}$. Est-il pour autant inexact de qualifier d' "experts" les prêtres de Rome? Il semble que non, Claudia Moatti qualifiant elle-même d' "expertise " l'action des pontifes, tout en précisant immédiatement qu'il s'agit d'une expertise particulière, "non seulement collective, mais secrète, et en tant que telle source de pouvoir " ${ }^{46}$. Ainsi, en dehors d'une certaine spécialisation en matière de droit sacré - mais cette spécialisation n'est jamais que relative, même pour les plus compétents -, les prêtres romains sont experts avant tout en vertu de leurs compétences générales d'aristocrates, habitués qu'ils sont à célébrer les cultes à titre privé comme à titre public, à débattre au Sénat des affaires religieuses, à pratiquer quotidiennement le droit en conseillant leurs clients ou en agissant en justice. Car, loin de se consacrer à leur seule fonction sacerdotale et à la seule étude du droit sacré, les prêtres romains sont d'abord, sous la République, des aristocrates qui gouvernent la cité. Au nom d'une conception de la cité comme "communauté des dieux et des hommes", ceux qui ont légitimité pour gouverner les hommes ont légitimité pour honorer les dieux.

43. La question de savoir quels étaient les prêtres inaugurés est discutée. Cf. P. CATALANO, Contributi allo studio del diritto augurale, Turin, 1960, t. I, p. 211-220.

44. C. MOATTI, «Experts, mémoire et pouvoir à Rome», art. cité, p. 309-310.

45. Ibid., p. 315-317 (en général), p. 317-319 (exemple des juristes) et p. 319-321 (exemple des antiquaires).

46. Ibid., p. 312. 